\title{
A convenient route to enantiomerically pure 2-substituted methyl glycerate derivatives
}

\author{
Steven V. Ley, ${ }^{*}$ Patrick Michel and Claudio Trapella \\ Department of Chemistry, University of Cambridge, Lensfield \\ Road, Cambridge CB2 1EW, UK \\ svl1000@cam.ac.uk
}

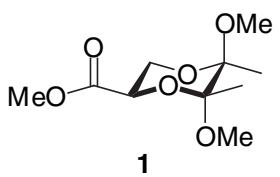

Boron trifluoride etherate $(10 \mathrm{~mL}, 81.3 \mathrm{mmol})$ was added to a stirred solution of D-mannitol $(61.9 \mathrm{~g}, 339.8 \mathrm{mmol})$, anhydrous trimethyl orthoformate (150 $\mathrm{mL}, 1.37 \mathrm{~mol})$ and butanedione $(63 \mathrm{~mL}, 717.8 \mathrm{mmol})$ in $\mathrm{MeOH}(300 \mathrm{~mL})$ at room temperature, under an atmosphere of argon. After $5 \mathrm{~h}$, the reaction mixture was neutralized by the addition of $\mathrm{Et}_{3} \mathrm{~N}(10 \mathrm{~mL}, 71.74 \mathrm{mmol})$ and the solvent was removed in vacuo. The residue was dissolved in $\mathrm{CH}_{2} \mathrm{Cl}_{2}(1.2 \mathrm{~L})$ and washed with water $(600 \mathrm{~mL})$ and brine $(300 \mathrm{~mL})$. The organic phase was dried $\left(\mathrm{Na}_{2} \mathrm{SO}_{4}\right)$, filtered and concentrated in vacuo. The crude residue was used without further purification. The BDAdiprotected D-mannitol could be purified by recrystallisation from hexane to give a white solid.

Mp $137-138^{\circ} \mathrm{C}$.

$[\alpha]_{\mathrm{D}}^{27}-222.7\left(\mathrm{c}=1.2, \mathrm{CHCl}_{3}\right)$.

IR: $3452,2946,1114 \mathrm{~cm}^{-1}$.

${ }^{1} \mathrm{H}$ NMR $\left(400 \mathrm{MHz}, \mathrm{CDCl}_{3}\right): \delta=4.07(2 \mathrm{H}, \mathrm{ddd}, J=11.0 \mathrm{~Hz}, 6.1 \mathrm{~Hz}, 3.6 \mathrm{~Hz}), 3.71(2 \mathrm{H}, \mathrm{dd}, J=$ $11.3 \mathrm{~Hz}, 11.3 \mathrm{~Hz}), 3.64(2 \mathrm{H}, \mathrm{dd}, J=11.4 \mathrm{~Hz}, 3.5 \mathrm{~Hz}), 3.26(6 \mathrm{H}, \mathrm{s}), 3.25(6 \mathrm{H}, \mathrm{s}), 2.78(2 \mathrm{H}, \mathrm{d}, J=$ $6.5 \mathrm{~Hz}), 1.26(6 \mathrm{H}, \mathrm{s}), 1.25(6 \mathrm{H}, \mathrm{s})$.

${ }^{13} \mathrm{C} \mathrm{NMR}\left(400 \mathrm{MHz}, \mathrm{CDCl}_{3}\right.$ ): $\delta=99.2,98.0,69.4,68.4,60.9,48.1,48.0,17.8,17.5$.

HRMS (+ESI): $m / z$ calcd for $\mathrm{C}_{18} \mathrm{H}_{34} \mathrm{NaO}_{10}\left(\mathrm{MNa}^{+}\right)$433.2050; found: 433.2040 .

Anal. Calcd for $\mathrm{C}_{18} \mathrm{H}_{34} \mathrm{O}_{10}$ : C, 52.67; H, 8.35. Found C, 52.57; H, 8.46.

Sodium metaperiodate $(95.4 \mathrm{~g}, 418.5 \mathrm{mmol})$ was added slowly to a stirred solution of the crude diol in $\mathrm{MeOH}(350 \mathrm{~mL})$ and water $(700 \mathrm{~mL})$ at $0^{\circ} \mathrm{C}$. After stirring overnight at room temperature, sodium hydrogenocarbonate $(109.6 \mathrm{~g}, 1.3 \mathrm{~mol})$ was added, followed by dropwise addition of bromine $(26 \mathrm{~mL}, 507.4 \mathrm{mmol})$ until a permanent yellow colour remained. Excess bromine was quenched by $\mathrm{Na}_{2} \mathrm{~S}_{2} \mathrm{O}_{3}$. The slurry was filtered and the filtrate was extracted with $\mathrm{CH}_{2} \mathrm{Cl}_{2}(2 \times 800$ $\mathrm{mL})$. The organic phases were washed with water $(400 \mathrm{~mL})$ and brine $(400 \mathrm{~mL})$, dried $\left(\mathrm{Na}_{2} \mathrm{SO}_{4}\right)$, filtered and concentrated in vacuo. The crude product was purified by fractional distillation using a Vigreux column (bp $92{ }^{\circ} \mathrm{C} / 0.11 \mathrm{mmHg}$ ) to afford 1 as a colourless oil $(73.5 \mathrm{~g}, 314.1 \mathrm{mmol}$, $46 \%)$.

$[\alpha]_{\mathrm{D}}^{25}-158.8\left(\mathrm{c}=1.22, \mathrm{CHCl}_{3}\right)$.

IR: $2953,1764,1735 \mathrm{~cm}^{-1}$. 
${ }^{1} \mathrm{H}$ NMR $\left(400 \mathrm{MHz}, \mathrm{CDCl}_{3}\right): \delta=4.53(1 \mathrm{H}, \mathrm{dd}, J=11.2 \mathrm{~Hz}, 7.6 \mathrm{~Hz}), 3.81(1 \mathrm{H}, \mathrm{dd}, J=11.2 \mathrm{~Hz}$, $11.2 \mathrm{~Hz}), 3.72-3.68(4 \mathrm{H}, \mathrm{m}), 3.28(3 \mathrm{H}, \mathrm{s}), 3.24(3 \mathrm{H}, \mathrm{s}), 1.35(3 \mathrm{H}, \mathrm{s}), 1.26(3 \mathrm{H}, \mathrm{s})$.

${ }^{13} \mathrm{C}$ NMR (400 MHz, $\mathrm{CDCl}_{3}$ ): $\delta=169.1,99.7,98.0,67.1,59.9,52.1,48.3,48.0,17.5,17.4$.

HRMS (EI): $\mathrm{m} / z$ calcd for $\mathrm{C}_{10} \mathrm{H}_{18} \mathrm{O}_{6}\left(\mathrm{M}^{+}\right)$234.1103; found: 234.1110 .

Anal. Calcd for $\mathrm{C}_{10} \mathrm{H}_{18} \mathrm{O}_{6}$ : C, 51.27; H, 7.75. Found C, 51.37; H, 7.80.

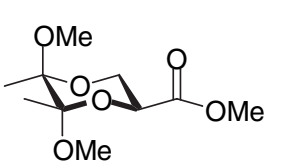

2

Boron trifluoride-tetrahydrofuran complex $(5 \mathrm{~mL}, 45.3 \mathrm{mmol})$ was added to a stirred solution of L-ascorbic acid (123.78 g, $702.8 \mathrm{mmol})$, anhydrous trimethyl orthoformate $(160 \mathrm{~mL}, 1.46 \mathrm{~mol})$ and butanedione $(60 \mathrm{~mL}, 683.7$ $\mathrm{mmol})$ in $\mathrm{MeOH}(300 \mathrm{~mL})$ at room temperature, under an atmosphere of argon. After 5h, the reaction mixture was neutralized by the addition of $\mathrm{Et}_{3} \mathrm{~N}$ $(6.5 \mathrm{~mL}, 46.63 \mathrm{mmol})$ and the solvent was removed in vacuo. The residue was dissolved in $\mathrm{CH}_{2} \mathrm{Cl}_{2}(1.2 \mathrm{~L})$ and washed with a solution of sodium chloride $(400 \mathrm{~mL}$ of water and $200 \mathrm{~mL}$ of brine). The organic phase was dried $\left(\mathrm{Na}_{2} \mathrm{SO}_{4}\right)$, filtered and concentrated in vacuo to give a white solid (184.4 g). The crude solid was used without further purification.

Hydrogen peroxide $35 \%(100 \mathrm{~mL}, 1.17 \mathrm{~mol})$ was added slowly $(1 \mathrm{~h})$ to a stirred solution of BDAprotected L-ascorbic acid and potassium carbonate $(149.3 \mathrm{~g}, 1.08 \mathrm{~mol})$ in water $(550 \mathrm{~mL})$ at ice bath temperature. The mixture was allowed to warm to room temperature and stirred overnight. $10 \%$ palladium on activated carbon $\left(500 \mathrm{mg}\right.$ ) was added and the mixture was heated to $45^{\circ} \mathrm{C}$ until a negative test to starch iodide paper was obtained $(1 \mathrm{~h})$. The reaction mixture was filtered through Celite and the solvent was removed in vacuo to give a white powder. Dimethyl sulfate $(70 \mathrm{~mL}$, $739.8 \mathrm{mmol}$ ) was added to a stirred suspension of the crude potassium carboxylate in acetone (500 $\mathrm{mL}$ ) at room temperature under an atmosphere of argon. The reaction mixture was stirred at reflux for $2 \mathrm{~h}$ and diluted with $\mathrm{Et}_{2} \mathrm{O}(1.2 \mathrm{~L})$. The organic phase was washed with water $(400 \mathrm{~mL})$, saturated aqueous solution of sodium hydrogenocarbonate $(400 \mathrm{~mL})$, dried $\left(\mathrm{Na}_{2} \mathrm{SO}_{4}\right)$, filtered and concentrated in vacuo to give a white solid $(112.73 \mathrm{~g})$. The crude solid was used without further purification.

Sodium borohydride $(24.08 \mathrm{~g}, 636.5 \mathrm{mmol})$ was added slowly to a stirred solution of previous crude ester in isopropanol $(400 \mathrm{~mL})$ at $20^{\circ} \mathrm{C}$, under an atmosphere of argon. The reaction mixture was heated to $60^{\circ} \mathrm{C}$ for $1 \mathrm{~h}$. Saturated aqueous ammonium chloride $(250 \mathrm{~mL})$ and water $(500 \mathrm{~mL})$ were slowly added to the solution. The aqueous phase was extracted with $\mathrm{CH}_{2} \mathrm{Cl}_{2}(750 \mathrm{~mL}, 2 \times 500$ $\mathrm{mL})$. The combined organic extracts were dried $\left(\mathrm{Na}_{2} \mathrm{SO}_{4}\right)$, filtered and concentrated in vacuo to give colourless oil $(83.23 \mathrm{~g})$. The crude residue was used without further purification.

Sodium metaperiodate $(93.1 \mathrm{~g}, 408.4 \mathrm{mmol})$ was added slowly to a stirred solution of the crude diol in $\mathrm{MeOH}(300 \mathrm{~mL})$ and water $(600 \mathrm{~mL})$ at room temperature. After stirring for $2 \mathrm{~h}$, sodium hydrogenocarbonate (112.2 g, $1.33 \mathrm{~mol}$ ) was added, followed by dropwise addition of bromine (32 $\mathrm{mL}, 624.5 \mathrm{mmol}$ ) until a permanent yellow colour remained. Excess bromine was quenched by $\mathrm{Na}_{2} \mathrm{~S}_{2} \mathrm{O}_{3}$. The slurry was filtered and the filtrate was extracted with $\mathrm{CH}_{2} \mathrm{Cl}_{2}(2 \times 800 \mathrm{~mL})$. The organic phases were washed with water $(400 \mathrm{~mL})$ and brine $(400 \mathrm{~mL})$, dried $\left(\mathrm{Na}_{2} \mathrm{SO}_{4}\right)$, filtered and concentrated in vacuo. The crude product was purified by fractional distillation using a Vigreux column (bp $92{ }^{\circ} \mathrm{C} / 0.11 \mathrm{mmHg}$ ) to afford 2 as a colourless oil $(60.1 \mathrm{~g}, 256.8 \mathrm{mmol}, 37 \%)$.

$[\alpha]_{\mathrm{D}}^{25}+161.5\left(\mathrm{c}=1.125, \mathrm{CHCl}_{3}\right)$.

Anal. Calcd for $\mathrm{C}_{10} \mathrm{H}_{18} \mathrm{O}_{6}$ : C, 51.27; H, 7.75. Found C, 51.29; H, 7.76. 


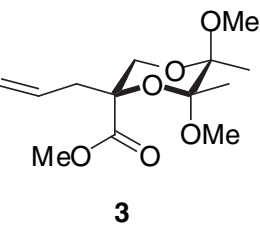

$n$-Butyllithium (3.95 $\mathrm{mL}, 2.5 \mathrm{M}$ in hexanes, $9.9 \mathrm{mmol})$ was added slowly to a stirred solution of diisopropylamine $(1.55 \mathrm{~mL}, 11.1 \mathrm{mmol})$ in THF $(5 \mathrm{~mL})$ at $-20{ }^{\circ} \mathrm{C}$ to $0{ }^{\circ} \mathrm{C}$. The solution was stirred for an additional $15 \mathrm{~min}$, then added via cannula to a stirred solution of $\mathbf{1}(1.54 \mathrm{~g}, 6.57 \mathrm{mmol})$ in THF (35 $\mathrm{mL})$ at $-78{ }^{\circ} \mathrm{C}$. After $10 \mathrm{~min}$, allyl iodide $(3.0 \mathrm{~mL}, 32.8 \mathrm{mmol})$ was added. The reaction mixture was stirred for $30 \mathrm{~min}$, then quenched at $-78{ }^{\circ} \mathrm{C}$ with saturated $\mathrm{NH}_{4} \mathrm{Cl}$ and diluted with $\mathrm{Et}_{2} \mathrm{O}(50 \mathrm{~mL})$. The organic phase was washed with saturated $\mathrm{NH}_{4} \mathrm{Cl}(50 \mathrm{~mL})$ and water $(50 \mathrm{~mL})$, dried $\left(\mathrm{Na}_{2} \mathrm{SO}_{4}\right)$, filtered and concentrated in vacuo. The residue was purified by flash chromatography (hexane-EtOAc 9:1) to give 3 as a white solid (1.1 g, $4.01 \mathrm{mmol}, 61 \%)$.

Mp 68-69 ${ }^{\circ} \mathrm{C}$

$[\alpha]_{D}^{26}-124.5\left(\mathrm{c}=1.52, \mathrm{CHCl}_{3}\right)$.

IR: $1735,1643 \mathrm{~cm}^{-1}$.

${ }^{1} \mathrm{H} \mathrm{NMR}\left(400 \mathrm{MHz}, \mathrm{CDCl}_{3}\right): \delta=5.65-5.53(1 \mathrm{H}, \mathrm{m}), 5.08-5.04(2 \mathrm{H}, \mathrm{m}), 4.11(1 \mathrm{H}, \mathrm{d}, J=11.7 \mathrm{~Hz})$, $3.75(3 \mathrm{H}, \mathrm{s}), 3.62(1 \mathrm{H}, \mathrm{d}, J=11.7 \mathrm{~Hz}), 3.25(3 \mathrm{H}, \mathrm{s}), 3.22(3 \mathrm{H}, \mathrm{s}), 2.44(1 \mathrm{H}, \mathrm{dd}, J=13.7 \mathrm{~Hz}, 7.7$ $\mathrm{Hz}), 2.37(1 \mathrm{H}, \mathrm{dd}, J=13.7 \mathrm{~Hz}, 7.2 \mathrm{~Hz}), 1.31(3 \mathrm{H}, \mathrm{s}), 1.27(3 \mathrm{H}, \mathrm{s})$.

${ }^{13} \mathrm{C}$ NMR $\left(400 \mathrm{MHz}, \mathrm{CDCl}_{3}\right): \delta=172.9,130.5,119.1,99.7,98.1,73.4,61.8,51.2,50.3,48.1$, 42.2, 17.8, 17.7 .

HRMS (+ESI): $m / z$ calcd for $\mathrm{C}_{13} \mathrm{H}_{22} \mathrm{NaO}_{6}\left(\mathrm{MNa}^{+}\right)$297.1314; found: 297.1327 .

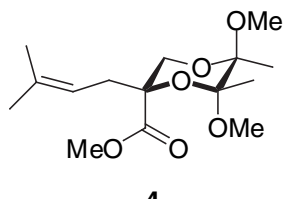

$n$-Butyllithium $(6 \mathrm{~mL}, 2.5 \mathrm{M}$ in hexanes, $15 \mathrm{mmol})$ was added slowly to a stirred solution of diisopropylamine $(2.4 \mathrm{~mL}, 17.1 \mathrm{mmol})$ in THF $(5 \mathrm{~mL})$ at $-20{ }^{\circ} \mathrm{C}$ to $0{ }^{\circ} \mathrm{C}$. The solution was stirred for an additional $15 \mathrm{~min}$, then added via cannula to a stirred solution of $\mathbf{1}(2.35 \mathrm{~g}, 10.05 \mathrm{mmol})$ in THF (45 $\mathrm{mL})$ at $-78{ }^{\circ} \mathrm{C}$. After $20 \mathrm{~min}$, 4-bromo-2-methyl-2-butene $(3.5 \mathrm{~mL}, 30.36$ mmol) was added. The reaction mixture was stirred for $30 \mathrm{~min}$, then quenched at $-78{ }^{\circ} \mathrm{C}$ with saturated $\mathrm{NH}_{4} \mathrm{Cl}$ and diluted with $\mathrm{Et}_{2} \mathrm{O}(50 \mathrm{~mL})$. The organic phase was washed with saturated $\mathrm{NH}_{4} \mathrm{Cl}(50 \mathrm{~mL})$ and water $(50 \mathrm{~mL})$, dried $\left(\mathrm{Na}_{2} \mathrm{SO}_{4}\right)$, filtered and concentrated in vacuo. The residue was purified by flash chromatography (hexane-EtOAc 4:1) to give $\mathbf{4}$ as a colourless oil (2.09 g, $6.93 \mathrm{mmol}, 69 \%)$.

$[\alpha]_{D}^{25}-95.7\left(\mathrm{c}=0.625, \mathrm{CHCl}_{3}\right)$.

IR: $1741 \mathrm{~cm}^{-1}$.

${ }^{1} \mathrm{H}$ NMR $\left(400 \mathrm{MHz}, \mathrm{CDCl}_{3}\right): \delta=4.85(1 \mathrm{H}, \mathrm{t}, J=11.7 \mathrm{~Hz}), 4.09(1 \mathrm{H}, \mathrm{d}, J=11.6 \mathrm{~Hz}), 3.70(3 \mathrm{H}, \mathrm{s})$, $3.55(1 \mathrm{H}, \mathrm{d}, J=11.6 \mathrm{~Hz}), 3.22(3 \mathrm{H}, \mathrm{s}), 3.18(3 \mathrm{H}, \mathrm{s}), 2.44(1 \mathrm{H}, \mathrm{dd}, J=11.6 \mathrm{~Hz}, 8.0 \mathrm{~Hz}), 2.26(1 \mathrm{H}$, $\mathrm{dd}, J=11.6 \mathrm{~Hz}, 7.2 \mathrm{~Hz}), 1.63(3 \mathrm{H}, \mathrm{s}), 1.54(3 \mathrm{H}, \mathrm{s}), 1.28(3 \mathrm{H}, \mathrm{s}), 1.23(3 \mathrm{H}, \mathrm{s})$.

${ }^{13} \mathrm{C}$ NMR $\left(400 \mathrm{MHz}, \mathrm{CDCl}_{3}\right): \delta=173.0,135.8,115.6,99.6,98.0,73.9,62.0,51.7,50.2,47.9$, 36.6, 25.8, 17.74, 17.73, 17.67.

HRMS (+ESI): $m / z$ calcd for $\mathrm{C}_{15} \mathrm{H}_{26} \mathrm{NaO}_{6}\left(\mathrm{MNa}^{+}\right)$325.1627; found: 325.1636 . 


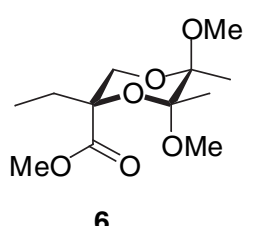

6

$n$-Butyllithium (1.6 mL, 2.5M in hexanes, $4 \mathrm{mmol})$ was added slowly to a stirred solution of diisopropylamine $(670 \mu \mathrm{L}, 4.8 \mathrm{mmol})$ in THF $(3 \mathrm{~mL})$ at $-20{ }^{\circ} \mathrm{C}$ to 0 ${ }^{\circ} \mathrm{C}$. The solution was stirred for an additional $15 \mathrm{~min}$, then added via cannula to a stirred solution of $1(479 \mathrm{mg}, 2.05 \mathrm{mmol})$ in THF $(10 \mathrm{~mL})$ at $-78{ }^{\circ} \mathrm{C}$. After 10 min, ethyl iodide $(820 \mu \mathrm{L}, 10.25 \mathrm{mmol})$ in HMPA $(1 \mathrm{~mL})$ was added. The reaction mixture was stirred for $30 \mathrm{~min}$, then quenched at $-78{ }^{\circ} \mathrm{C}$ with saturated $\mathrm{NH}_{4} \mathrm{Cl}$ and diluted with $\mathrm{Et}_{2} \mathrm{O}(20 \mathrm{~mL})$. The organic phase was washed with saturated $\mathrm{NH}_{4} \mathrm{Cl}(20$ $\mathrm{mL})$ and water $(20 \mathrm{~mL})$, dried $\left(\mathrm{Na}_{2} \mathrm{SO}_{4}\right)$, filtered and concentrated in vacuo. The residue was purified by flash chromatography (hexane-EtOAc 4:1) to give $\mathbf{6}$ as a colourless oil (320 mg, 1.22 mmol, 60\%).

$[\alpha]_{D}^{25}-90.9\left(\mathrm{c}=1.25, \mathrm{CHCl}_{3}\right)$.

IR: $1739 \mathrm{~cm}^{-1}$.

${ }^{1} \mathrm{H}$ NMR $\left(400 \mathrm{MHz}, \mathrm{CDCl}_{3}\right): \delta=4.12(1 \mathrm{H}, \mathrm{d}, J=11.5 \mathrm{~Hz}), 3.72(3 \mathrm{H}, \mathrm{s}), 3.53(1 \mathrm{H}, \mathrm{d}, J=11.5$ $\mathrm{Hz}), 3.20(3 \mathrm{H}, \mathrm{s}), 3.17(3 \mathrm{H}, \mathrm{s}), 1.73-1.53(2 \mathrm{H}, \mathrm{m}), 1.25(3 \mathrm{H}, \mathrm{s}), 1.22(3 \mathrm{H}, \mathrm{s}), 0.73(3 \mathrm{H}, \mathrm{t}, J=7.7$ $\mathrm{Hz}$.

${ }^{13} \mathrm{C}$ NMR $\left(400 \mathrm{MHz}, \mathrm{CDCl}_{3}\right): \delta=173.2,99.4,98.0,74.0,61.8,51.7,50.2,47.9,30.5,17.7,17.6$, 7.1.

HRMS (+ESI): $m / z$ calcd for $\mathrm{C}_{12} \mathrm{H}_{22} \mathrm{NaO}_{6}\left(\mathrm{MNa}^{+}\right)$285.1314; found: 285.1319 .

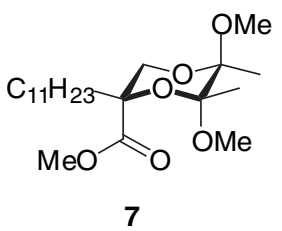

$n$-Butyllithium ( $5 \mathrm{~mL}, 2.5 \mathrm{M}$ in hexanes, $12.5 \mathrm{mmol}$ ) was added slowly to a stirred solution of diisopropylamine $(2.1 \mathrm{~mL}, 15 \mathrm{mmol})$ in THF $(5 \mathrm{~mL})$ at -20 ${ }^{\circ} \mathrm{C}$ to $0{ }^{\circ} \mathrm{C}$. The solution was stirred for an additional $15 \mathrm{~min}$, then added via cannula to a stirred solution of $\mathbf{1}(2.08 \mathrm{~g}, 8.89 \mathrm{mmol})$ in THF $(35 \mathrm{~mL})$ at -78 ${ }^{\circ} \mathrm{C}$. After $30 \mathrm{~min}$, 1-iodoundecane $(6.2 \mathrm{~mL}, 26.8 \mathrm{mmol})$ in HMPA $(4 \mathrm{~mL})$ was added. The reaction mixture was stirred for $1 \mathrm{~h}$, then quenched at $-78^{\circ} \mathrm{C}$ with saturated $\mathrm{NH}_{4} \mathrm{Cl}$ and diluted with $\mathrm{Et}_{2} \mathrm{O}(50 \mathrm{~mL})$. The organic phase was washed with saturated $\mathrm{NH}_{4} \mathrm{Cl}(50 \mathrm{~mL})$ and water $(50 \mathrm{~mL})$, dried $\left(\mathrm{Na}_{2} \mathrm{SO}_{4}\right)$, filtered and concentrated in vacuo. The residue was purified by flash chromatography (hexane-EtOAc 9:1) to give 7 as a colourless oil $(1.90 \mathrm{~g}, 4.9 \mathrm{mmol}, 55 \%)$.

$[\alpha]_{D}^{25}-210.5\left(\mathrm{c}=0.92, \mathrm{CHCl}_{3}\right)$.

IR: $2923,1742 \mathrm{~cm}^{-1}$.

${ }^{1} \mathrm{H}$ NMR $\left(400 \mathrm{MHz}, \mathrm{CDCl}_{3}\right): \delta=4.12(1 \mathrm{H}, \mathrm{d}, J=11.5 \mathrm{~Hz}), 3.72(3 \mathrm{H}, \mathrm{s}), 3.55(1 \mathrm{H}, \mathrm{d}, J=11.5$ $\mathrm{Hz}), 3.21(3 \mathrm{H}, \mathrm{s}), 3.18(3 \mathrm{H}, \mathrm{s}), 1.69-1.49(2 \mathrm{H}, \mathrm{m}), 1.27-1.10(24 \mathrm{H}, \mathrm{m}), 0.85-0.81(3 \mathrm{H}, \mathrm{m})$.

${ }^{13} \mathrm{C}$ NMR $\left(400 \mathrm{MHz}, \mathrm{CDCl}_{3}\right): \delta=173.0,99.1,97.8,73.5,61.9,51.4,49.9,47.6,37.4,31.6,29.5$, 29.2 (2C), 29.1, 29.0, 28.9, 22.31, 22.27, 17.43, 17.41, 13.7.

HRMS (+ESI): $\mathrm{m} / z$ calcd for $\mathrm{C}_{21} \mathrm{H}_{40} \mathrm{NaO}_{6}\left(\mathrm{MNa}^{+}\right)$411.2723; found: 411.2739 .

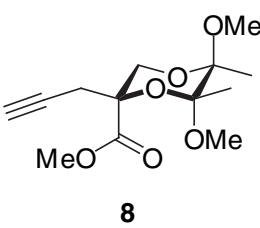

$n$-Butyllithium (1.2 $\mathrm{mL}, 2.5 \mathrm{M}$ in hexanes, $3 \mathrm{mmol})$ was added slowly to a stirred solution of diisopropylamine $(480 \mu \mathrm{L}, 3.42 \mathrm{mmol})$ in THF $(3 \mathrm{~mL})$ at $-20{ }^{\circ} \mathrm{C}$ to $0{ }^{\circ} \mathrm{C}$. The solution was stirred for an additional $15 \mathrm{~min}$, then added via cannula to a stirred solution of $\mathbf{1}(470 \mathrm{mg}, 2.01 \mathrm{mmol})$ in THF $(10 \mathrm{~mL})$ at $-78{ }^{\circ} \mathrm{C}$. After $10 \mathrm{~min}$, propargyl bromide $(900 \mu \mathrm{L}, 10.1 \mathrm{mmol})$ in HMPA 
$(1 \mathrm{~mL})$ was added. The reaction mixture was stirred for $1 \mathrm{~h}$, then quenched at $-78{ }^{\circ} \mathrm{C}$ with saturated $\mathrm{NH}_{4} \mathrm{Cl}$ and diluted with $\mathrm{Et}_{2} \mathrm{O}(30 \mathrm{~mL})$. The organic phase was washed with saturated $\mathrm{NH}_{4} \mathrm{Cl}(30 \mathrm{~mL})$ and water $(20 \mathrm{~mL})$, dried $\left(\mathrm{Na}_{2} \mathrm{SO}_{4}\right)$, filtered and concentrated in vacuo. The residue was purified by flash chromatography (hexane-EtOAc 9:1) to give 8 as a white solid (308 $\mathrm{mg}, 1.13 \mathrm{mmol}, 56 \%) .8$ could be recrystallised from hexane.

$\operatorname{Mp} 102^{\circ} \mathrm{C}$.

$[\alpha]_{\mathrm{D}}^{25}-159.9\left(\mathrm{c}=0.885, \mathrm{CHCl}_{3}\right)$.

IR: $3263,1742 \mathrm{~cm}^{-1}$.

${ }^{1} \mathrm{H}$ NMR $\left(400 \mathrm{MHz}, \mathrm{CDCl}_{3}\right): \delta=4.29(1 \mathrm{H}, \mathrm{d}, J=11.7 \mathrm{~Hz}), 3.75(3 \mathrm{H}, \mathrm{s}), 3.64(1 \mathrm{H}, \mathrm{d}, J=11.7$ $\mathrm{Hz}), 3.22(3 \mathrm{H}, \mathrm{s}), 3.16(3 \mathrm{H}, \mathrm{s}), 2.62(1 \mathrm{H}, \mathrm{dd}, J=16.5 \mathrm{~Hz}, 2.8 \mathrm{~Hz}), 2.41(1 \mathrm{H}, \mathrm{dd}, J=16.5 \mathrm{~Hz}, 2.7$ $\mathrm{Hz}), 2.00(1 \mathrm{H}, \mathrm{t}, J=2.7 \mathrm{~Hz}), 1.25(3 \mathrm{H}, \mathrm{s}), 1.23(3 \mathrm{H}, \mathrm{s})$.

${ }^{13} \mathrm{C}$ NMR $\left(400 \mathrm{MHz}, \mathrm{CDCl}_{3}\right): \delta=171.8,99.8,98.0,76.5,72.6,72.1,61.7,52.0,50.1,48.0,27.6$, 17.6, 17.5 .

HRMS (+ESI): $m / z$ calcd for $\mathrm{C}_{13} \mathrm{H}_{20} \mathrm{NaO}_{6}\left(\mathrm{MNa}^{+}\right)$295.1158; found: 295.1159 .

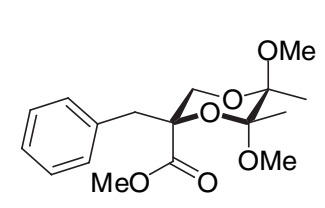

9

$n$-Butyllithium ( $1.2 \mathrm{~mL}, 2.5 \mathrm{M}$ in hexanes, $3 \mathrm{mmol})$ was added slowly to a stirred solution of diisopropylamine $(480 \mu \mathrm{L}, 3.42 \mathrm{mmol})$ in THF $(3 \mathrm{~mL})$ at $-20{ }^{\circ} \mathrm{C}$ to $0{ }^{\circ} \mathrm{C}$. The solution was stirred for an additional $15 \mathrm{~min}$, then added via cannula to a stirred solution of $1(461 \mathrm{mg}, 1.97 \mathrm{mmol})$ in THF $(10 \mathrm{~mL})$ at $-78{ }^{\circ} \mathrm{C}$. After $10 \mathrm{~min}$, benzyl bromide $(1.2 \mathrm{~mL}, 10.08 \mathrm{mmol})$ in HMPA $(1 \mathrm{~mL})$ was added. The reaction mixture was stirred for $1 \mathrm{~h}$, then quenched at $-78{ }^{\circ} \mathrm{C}$ with saturated $\mathrm{NH}_{4} \mathrm{Cl}$ and diluted with $\mathrm{Et}_{2} \mathrm{O}(30 \mathrm{~mL})$. The organic phase was washed with saturated $\mathrm{NH}_{4} \mathrm{Cl}(30 \mathrm{~mL})$ and water $(20 \mathrm{~mL})$, dried $\left(\mathrm{Na}_{2} \mathrm{SO}_{4}\right)$, filtered and concentrated in vacuo. The residue was purified by flash chromatography (hexane-EtOAc 9:1) to give 9 as a colourless oil (433 mg, $1.34 \mathrm{mmol}, 68 \%)$.

$[\alpha]_{D}^{25}-118.8\left(\mathrm{c}=0.835, \mathrm{CHCl}_{3}\right)$.

IR: $1739 \mathrm{~cm}^{-1}$.

${ }^{1} \mathrm{H}$ NMR $\left(400 \mathrm{MHz}, \mathrm{CDCl}_{3}\right): \delta=7.22-7.04(5 \mathrm{H}, \mathrm{m}), 3.95(1 \mathrm{H}, \mathrm{d}, J=11.6 \mathrm{~Hz}), 3.69(1 \mathrm{H}, \mathrm{d}, J=$ $11.6 \mathrm{~Hz}), 3.61(3 \mathrm{H}, \mathrm{s}), 3.20(3 \mathrm{H}, \mathrm{s}), 3.19(3 \mathrm{H}, \mathrm{s}), 3.00(1 \mathrm{H}, \mathrm{d}, J=13.3 \mathrm{~Hz}), 2.91(1 \mathrm{H}, \mathrm{d}, J=13.3$ $\mathrm{Hz}), 1.32(3 \mathrm{H}, \mathrm{s}), 1.23(3 \mathrm{H}, \mathrm{s})$.

${ }^{13} \mathrm{C}$ NMR (400 MHz, $\mathrm{CDCl}_{3}$ ): $\delta=172.4,134.0,129.7$ (2C), 128.0 (2C), 126.9, 99.7, 97.9, 74.2, 61.8, 51.5, 50.1, 47.8, 43.9, 17.64, 17.60.

HRMS (+ESI): $m / z$ calcd for $\mathrm{C}_{17} \mathrm{H}_{24} \mathrm{NaO}_{6}\left(\mathrm{MNa}^{+}\right)$347.1471; found: 347.1487 .

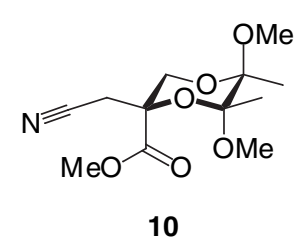

$n$-Butyllithium (1.9 mL, $1.6 \mathrm{M}$ in hexanes, $3.04 \mathrm{mmol})$ was added slowly to a stirred solution of diisopropylamine $(480 \mu \mathrm{L}, 3.42 \mathrm{mmol})$ in THF $(3 \mathrm{~mL})$ at $-20{ }^{\circ} \mathrm{C}$ to $0{ }^{\circ} \mathrm{C}$. The solution was stirred for an additional $15 \mathrm{~min}$, then added via cannula to a stirred solution of $\mathbf{1}(474 \mathrm{mg}, 2.02 \mathrm{mmol})$ in THF (10 $\mathrm{mL})$ at $-78{ }^{\circ} \mathrm{C}$. After $10 \mathrm{~min}$, bromoacetonitrile $(700 \mu \mathrm{L}, 10.05 \mathrm{mmol})$ in HMPA $(1 \mathrm{~mL})$ was added. The reaction mixture was stirred for $1 \mathrm{~h}$, then quenched at $-78{ }^{\circ} \mathrm{C}$ with saturated $\mathrm{NH}_{4} \mathrm{Cl}$ and diluted with $\mathrm{Et}_{2} \mathrm{O}(30 \mathrm{~mL})$. The organic phase was 
washed with saturated $\mathrm{NH}_{4} \mathrm{Cl}(30 \mathrm{~mL})$ and water $(20 \mathrm{~mL})$, dried $\left(\mathrm{Na}_{2} \mathrm{SO}_{4}\right)$, filtered and concentrated in vacuo. The residue was purified by flash chromatography (hexane-EtOAc 4:1) to give 10 as a colourless oil (308 $\mathrm{mg}, 1.13 \mathrm{mmol}, 56 \%)$.

$[\alpha]_{D}^{25}-111.9\left(\mathrm{c}=0.78, \mathrm{CHCl}_{3}\right)$.

IR: $1741 \mathrm{~cm}^{-1}$.

${ }^{1} \mathrm{H}$ NMR $\left(400 \mathrm{MHz}, \mathrm{CDCl}_{3}\right): \delta=4.25(1 \mathrm{H}, \mathrm{d}, J=11.5 \mathrm{~Hz}), 3.82(3 \mathrm{H}, \mathrm{s}), 3.77(1 \mathrm{H}, \mathrm{d}, J=11.5$

$\mathrm{Hz}), 3.27(3 \mathrm{H}, \mathrm{s}), 3.20(3 \mathrm{H}, \mathrm{s}), 2.66(2 \mathrm{H}, \mathrm{s}), 1.31(3 \mathrm{H}, \mathrm{s}), 1.27(3 \mathrm{H}, \mathrm{s})$.

${ }^{13} \mathrm{C}$ NMR $\left(400 \mathrm{MHz}, \mathrm{CDCl}_{3}\right): \delta=170.9,114.5,100.4,98.1,70.9,60.9,52.8,50.4,48.2,26.0$, 17.6, 17.5.

HRMS (+ESI): $m / z$ calcd for $\mathrm{C}_{12} \mathrm{H}_{19} \mathrm{NNaO}_{6}\left(\mathrm{MNa}^{+}\right)$296.1110; found: 296.1107 .

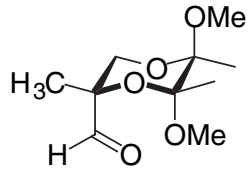

11

Lithium aluminium hydride $(52.7 \mathrm{~mL}, 1 \mathrm{M}$ in THF, $52.7 \mathrm{mmol})$ was added slowly to a stirred solution of $\mathbf{5}(18.43 \mathrm{~g}, 74.3 \mathrm{mmol})$ in $\mathrm{THF}(300 \mathrm{~mL})$ at $0{ }^{\circ} \mathrm{C}$ under argon. The mixture was stirred overnight at room temperature. Excess hydride was quenched by successive addition of water $(2 \mathrm{~mL}), 15 \%$ aqueous $\mathrm{NaOH}(2 \mathrm{~mL})$ and water $(6 \mathrm{~mL})$. The resulting suspension was filtered through Celite, rinsed with $\mathrm{Et}_{2} \mathrm{O}$, and the solvents were removed in vacuo. The crude residue was used without further purification.

DMSO (10.7 mL, $150.8 \mathrm{mmol})$ was added dropwise to a stirred solution pf oxalyl chloride (6.3 $\mathrm{mL}, 72.2 \mathrm{mmol})$ in $\mathrm{CH}_{2} \mathrm{Cl}_{2}(220 \mathrm{~mL})$ at $-50{ }^{\circ} \mathrm{C}$ to $-60{ }^{\circ} \mathrm{C}$, under argon. After $2 \mathrm{~min}$, a solution of the crude alcohol $(14.39 \mathrm{~g})$ in $\mathrm{CH}_{2} \mathrm{Cl}_{2}(20 \mathrm{~mL})$ was added. After stirring for $15 \mathrm{~min}, \mathrm{Et}_{3} \mathrm{~N}(29 \mathrm{~mL}$, $208 \mathrm{mmol}$ ) was added. The mixture was stirred for $5 \mathrm{~min}$ and then allowed to warm to room temperature. Water $(250 \mathrm{~mL})$ was added and the aqueous layer was extracted with $\mathrm{CH}_{2} \mathrm{Cl}_{2}(250$ $\mathrm{mL})$. The organic layers were combined, washed with brine $(500 \mathrm{~mL}), 1 \mathrm{~N} \mathrm{HCl}$ solution $(500 \mathrm{~mL})$, water $(250 \mathrm{~mL})$, saturated $\mathrm{NaHCO}_{3}(500 \mathrm{~mL})$, dried $\left(\mathrm{Na}_{2} \mathrm{SO}_{4}\right)$, filtered and concentrated in vacuo. The residue was purified by flash chromatography (hexane-EtOAc 9:1) to give $\mathbf{1 1}$ as a white solid (9.76 g, $44.8 \mathrm{mmol}, 60 \%)$. 11 could be recrystallised from hexane.

Mp 63-64 ${ }^{\circ} \mathrm{C}$.

$[\alpha]_{\mathrm{D}}^{25}-150.7\left(\mathrm{c}=1.265, \mathrm{CHCl}_{3}\right)$.

IR: $1725 \mathrm{~cm}^{-1}$.

${ }^{1} \mathrm{H}$ NMR $\left(400 \mathrm{MHz}, \mathrm{CDCl}_{3}\right): \delta=9.46(1 \mathrm{H}, \mathrm{d}, J=1.9 \mathrm{~Hz}), 4.02(1 \mathrm{H}, \mathrm{d}, J=11.4 \mathrm{~Hz}), 3.54(1 \mathrm{H}, \mathrm{dd}$, $J=11.4 \mathrm{~Hz}, 1.9 \mathrm{~Hz}), 3.36(3 \mathrm{H}, \mathrm{s}), 3.26(3 \mathrm{H}, \mathrm{s}), 1.35(3 \mathrm{H}, \mathrm{s}), 1.24(3 \mathrm{H}, \mathrm{s}), 1.04(3 \mathrm{H}, \mathrm{s})$.

${ }^{13} \mathrm{C}$ NMR $\left(400 \mathrm{MHz}, \mathrm{CDCl}_{3}\right): \delta=201.3,99.8,97.9,75.5,60.9,50.2,48.1,18.5,17.8,17.6$.

HRMS (+ESI): $m / z$ calcd for $\mathrm{C}_{10} \mathrm{H}_{18} \mathrm{NaO}_{5}\left(\mathrm{MNa}^{+}\right)$241.1052; found: 241.1043 .

Anal. Calcd for $\mathrm{C}_{10} \mathrm{H}_{18} \mathrm{O}_{5}$ : C, 55.03; H, 8.31. Found C, 54.96; H, 8.33.

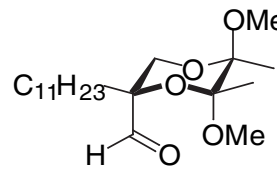

12
Lithium aluminium hydride (13.17 $\mathrm{mL}, 1 \mathrm{M}$ in $\mathrm{THF}, 13.17 \mathrm{mmol}$ ) was added slowly to a stirred solution of 7 (6.17 g, $15.9 \mathrm{mmol})$ in THF (100 $\mathrm{mL}$ ) at $0{ }^{\circ} \mathrm{C}$ under argon. The mixture was stirred overnight at room temperature. Excess hydride was quenched by successive addition of water $(0.5 \mathrm{~mL}), 15 \%$ aqueous $\mathrm{NaOH}(0.5 \mathrm{~mL})$ and water $(1.5 \mathrm{~mL})$. The resulting suspension was filtered through Celite, rinsed with $\mathrm{Et}_{2} \mathrm{O}$, and the solvents 
were removed in vacuo. The crude residue was used without further purification.

DMSO $(2.4 \mathrm{~mL}, 33.8 \mathrm{mmol})$ was added dropwise to a stirred solution pf oxalyl chloride $(1.4 \mathrm{~mL}$, $16.07 \mathrm{mmol})$ in $\mathrm{CH}_{2} \mathrm{Cl}_{2}(100 \mathrm{~mL})$ at $-50{ }^{\circ} \mathrm{C}$ to $-60{ }^{\circ} \mathrm{C}$, under argon. After $2 \mathrm{~min}$, a solution of the crude alcohol $(5.26 \mathrm{~g})$ in $\mathrm{CH}_{2} \mathrm{Cl}_{2}(10 \mathrm{~mL})$ was added. After stirring for $15 \mathrm{~min}, \mathrm{Et}_{3} \mathrm{~N}(6.5 \mathrm{~mL}, 46.6$ mmol) was added. The mixture was stirred for $5 \mathrm{~min}$ and then allowed to warm to room temperature. Water $(100 \mathrm{~mL})$ was added and the aqueous layer was extracted with $\mathrm{CH}_{2} \mathrm{Cl}_{2}(100$ $\mathrm{mL})$. The organic layers were combined, washed with brine $(200 \mathrm{~mL}), 1 \mathrm{~N} \mathrm{HCl}$ solution $(200 \mathrm{~mL})$, water $(100 \mathrm{~mL})$, saturated $\mathrm{NaHCO}_{3}(200 \mathrm{~mL})$, dried $\left(\mathrm{Na}_{2} \mathrm{SO}_{4}\right)$, filtered and concentrated in vacuo. The residue was purified by flash chromatography (hexane-EtOAc 9:1) to give $\mathbf{1 1}$ as a pale yellow solid (solidifies on refrigeration, $4.07 \mathrm{~g}, 11.37 \mathrm{mmol}, 71 \%$ ).

$[\alpha]_{\mathrm{D}}^{26}-73.8\left(\mathrm{c}=2.25, \mathrm{CHCl}_{3}\right)$.

IR: $1725 \mathrm{~cm}^{-1}$.

${ }^{1} \mathrm{H}$ NMR $\left(400 \mathrm{MHz}, \mathrm{CDCl}_{3}\right): \delta=9.43(1 \mathrm{H}, \mathrm{d}, J=1.7 \mathrm{~Hz}), 4.00(1 \mathrm{H}, \mathrm{d}, J=11.3 \mathrm{~Hz}), 3.50(1 \mathrm{H}, \mathrm{dd}$, $J=11.3 \mathrm{~Hz}, 1.7 \mathrm{~Hz}), 3.33(3 \mathrm{H}, \mathrm{s}), 3.23(3 \mathrm{H}, \mathrm{s}), 1.50-1.00(26 \mathrm{H}, \mathrm{m}), 0.85(3 \mathrm{H}, \mathrm{t}, J=6.8 \mathrm{~Hz})$.

${ }^{13} \mathrm{C}$ NMR $\left(400 \mathrm{MHz}, \mathrm{CDCl}_{3}\right): \delta=202.2,99.5,98.0,77.4,60.0,50.1,48.0,33.5,31.8,29.9,29.48$, $29.47,29.38,29.22,29.15,22.6,21.6,17.7,17.6,14.0$.

HRMS (+ESI): $\mathrm{m} / z$ calcd for $\mathrm{C}_{20} \mathrm{H}_{38} \mathrm{NaO}_{5}\left(\mathrm{MNa}^{+}\right)$381.2617; found: 381.2618 .

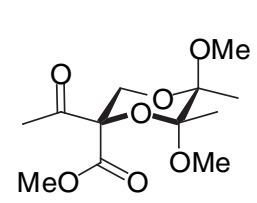

13

$n$-Butyllithium $(2.7 \mathrm{~mL}, 2.65 \mathrm{M}$ in heptane, $7.2 \mathrm{mmol})$ was added slowly to a stirred solution of diisopropylamine $(1.15 \mathrm{~mL}, 8.2 \mathrm{mmol})$ in THF $(4 \mathrm{~mL})$ at $-20{ }^{\circ} \mathrm{C}$ to $0{ }^{\circ} \mathrm{C}$. The solution was stirred for an additional $15 \mathrm{~min}$, then added via cannula to a stirred solution of $\mathbf{1}(1.12 \mathrm{~g}, 4.78 \mathrm{mmol})$ in THF $(36 \mathrm{~mL})$ at $-78{ }^{\circ} \mathrm{C}$. After $10 \mathrm{~min}$, acetyl chloride $(1.7 \mathrm{~mL}, 32.8 \mathrm{mmol})$ was added. The reaction mixture was stirred for $1 \mathrm{~h}$, then quenched at $-78{ }^{\circ} \mathrm{C}$ with saturated $\mathrm{NH}_{4} \mathrm{Cl}$ and diluted with $\mathrm{Et}_{2} \mathrm{O}(40 \mathrm{~mL})$. The organic phase was washed with saturated $\mathrm{NH}_{4} \mathrm{Cl}(40$ $\mathrm{mL})$ and water $(40 \mathrm{~mL})$, dried $\left(\mathrm{Na}_{2} \mathrm{SO}_{4}\right)$, filtered and concentrated in vacuo. The residue was purified by flash chromatography (hexane-EtOAc 4:1) to give $\mathbf{1 3}$ as a white solid (754 $\mathrm{mg}, 2.73$ $\mathrm{mmol}, 57 \%) .13$ could be recrystallised from hexane.

$n$-Butyllithium ( $3.8 \mathrm{~mL}, 2.65 \mathrm{M}$ in heptane, $10.07 \mathrm{mmol})$ was added slowly to a stirred solution of diisopropylamine $(1.6 \mathrm{~mL}, 11.4 \mathrm{mmol})$ in THF $(4 \mathrm{~mL})$ at $-20{ }^{\circ} \mathrm{C}$ to $0{ }^{\circ} \mathrm{C}$. The solution was stirred for an additional $15 \mathrm{~min}$, then added via cannula to a stirred solution of $1(1.58 \mathrm{~g}, 6.75 \mathrm{mmol})$ in THF $(36 \mathrm{~mL})$ at $-78{ }^{\circ} \mathrm{C}$. After $10 \mathrm{~min}$, acetic anhydride $(3.2 \mathrm{~mL}, 33.8 \mathrm{mmol})$ was added. The reaction mixture was stirred for $1 \mathrm{~h}$, then quenched at $-78^{\circ} \mathrm{C}$ with saturated $\mathrm{NH}_{4} \mathrm{Cl}$ and diluted with $\mathrm{Et}_{2} \mathrm{O}(40 \mathrm{~mL})$. The organic phase was washed with saturated $\mathrm{NH}_{4} \mathrm{Cl}(40 \mathrm{~mL})$ and water $(40$ $\mathrm{mL})$, dried $\left(\mathrm{Na}_{2} \mathrm{SO}_{4}\right)$, filtered and concentrated in vacuo. The residue was purified by flash chromatography (hexane-EtOAc 4:1) to give $\mathbf{1 3}$ as a white solid (1.19 mg, $4.31 \mathrm{mmol}, 64 \%$ ).

Mp $81-83{ }^{\circ} \mathrm{C}$.

$[\alpha]_{\mathrm{D}}^{25}-90.5\left(\mathrm{c}=1.12, \mathrm{CHCl}_{3}\right)$.

IR: $1750,1716 \mathrm{~cm}^{-1}$.

${ }^{1} \mathrm{H}$ NMR $\left(400 \mathrm{MHz}, \mathrm{CDCl}_{3}\right): \delta=4.20(1 \mathrm{H}, \mathrm{d}, J=11.6 \mathrm{~Hz}), 3.76(3 \mathrm{H}, \mathrm{s}), 3.75(1 \mathrm{H}, \mathrm{d}, J=11.6$ $\mathrm{Hz}), 3.27(3 \mathrm{H}, \mathrm{s}), 3.20(3 \mathrm{H}, \mathrm{s}), 2.31(3 \mathrm{H}, \mathrm{s}), 1.38(3 \mathrm{H}, \mathrm{s}), 1.27(3 \mathrm{H}, \mathrm{s})$.

${ }^{13} \mathrm{C}$ NMR (400 MHz, $\mathrm{CDCl}_{3}$ ): $\delta=204.7,168.7,100.2,98.2,80.6,58.7,52.7,50.1,48.0,25.3,17.8$ (2C).

HRMS (+ESI): $\mathrm{m} / z$ calcd for $\mathrm{C}_{12} \mathrm{H}_{20} \mathrm{NaO}_{7}\left(\mathrm{MNa}^{+}\right)$299.1107; found: 299.1116 . 


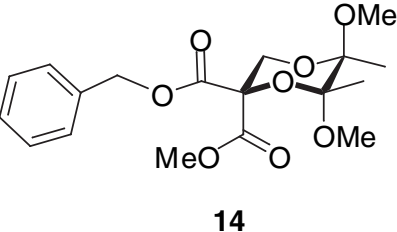

$n$-Butyllithium $(2.9 \mathrm{~mL}, 2.5 \mathrm{M}$ in hexanes, $7.25 \mathrm{mmol})$ was added slowly to a stirred solution of diisopropylamine $(1.1 \mathrm{~mL}, 7.85$ mmol) in THF ( $4 \mathrm{~mL})$ at $-20{ }^{\circ} \mathrm{C}$ to $0{ }^{\circ} \mathrm{C}$. The solution was stirred for an additional $15 \mathrm{~min}$, then added via cannula to a stirred solution of $1(1.12 \mathrm{~g}, 4.78 \mathrm{mmol})$ in THF $(36 \mathrm{~mL})$ at $-78{ }^{\circ} \mathrm{C}$. After $10 \mathrm{~min}$, benzyl chloroformate $(3.4 \mathrm{~mL}, 23.8 \mathrm{mmol})$ was added. The reaction mixture was stirred for $1 \mathrm{~h}$, then quenched at $-78{ }^{\circ} \mathrm{C}$ with saturated $\mathrm{NH}_{4} \mathrm{Cl}$ and diluted with $\mathrm{Et}_{2} \mathrm{O}(30 \mathrm{~mL})$. The organic phase was washed with saturated $\mathrm{NH}_{4} \mathrm{Cl}(30 \mathrm{~mL})$ and water $(30 \mathrm{~mL})$, dried $\left(\mathrm{Na}_{2} \mathrm{SO}_{4}\right)$, filtered and concentrated in vacuo. The residue was purified by flash chromatography (hexane-EtOAc 9:1) to give $\mathbf{1 4}$ as a pale yellow oil (1.08 g, $2.94 \mathrm{mmol}, 62 \%)$.

$[\alpha]_{\mathrm{D}}^{25}-55.0\left(\mathrm{c}=1.12, \mathrm{CHCl}_{3}\right)$.

IR: $1749 \mathrm{~cm}^{-1}$.

${ }^{1} \mathrm{H}$ NMR $\left(400 \mathrm{MHz}, \mathrm{CDCl}_{3}\right): \delta=7.35-7.20(5 \mathrm{H}, \mathrm{m}), 5.22(1 \mathrm{H}, \mathrm{d}, J=12.3 \mathrm{~Hz}), 5.07(1 \mathrm{H}, \mathrm{d}, J=$ $12.3 \mathrm{~Hz}), 4.28(1 \mathrm{H}, \mathrm{d}, J=11.4 \mathrm{~Hz}), 3.80(1 \mathrm{H}, \mathrm{d}, J=11.4 \mathrm{~Hz}), 3.67(3 \mathrm{H}, \mathrm{s}), 3.23(3 \mathrm{H}, \mathrm{s}), 3.17(3 \mathrm{H}$, $\mathrm{s}), 1.38(3 \mathrm{H}, \mathrm{s}), 1.24(3 \mathrm{H}, \mathrm{s})$.

${ }^{13} \mathrm{C} \mathrm{NMR}\left(400 \mathrm{MHz}, \mathrm{CDCl}_{3}\right): \delta=168.2,166.0,134.9,128.4(2 \mathrm{C}), 128.3,127.9$ (2C), 100.3, 98.1, 75.9, 67.3, 59.3, 52.5, 50.1, 48.0, 17.6, 17.5.

HRMS (+ESI): $\mathrm{m} / z$ calcd for $\mathrm{C}_{18} \mathrm{H}_{24} \mathrm{NaO}_{8}\left(\mathrm{MNa}^{+}\right)$391.1369; found: 391.1365 .

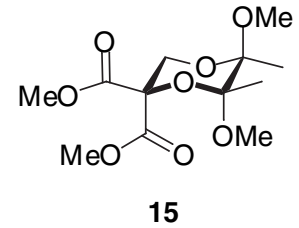
with saturated $\mathrm{NH}_{4} \mathrm{Cl}$ and diluted with $\mathrm{Et}_{2} \mathrm{O}(40 \mathrm{~mL})$. The organic phase was washed with saturated $\mathrm{NH}_{4} \mathrm{Cl}(40 \mathrm{~mL})$ and water $(40 \mathrm{~mL})$, dried $\left(\mathrm{Na}_{2} \mathrm{SO}_{4}\right)$, filtered and concentrated in vacuo. The residue was purified by flash chromatography (hexane-EtOAc 1:1) to give $\mathbf{1 5}$ as a white solid (1.31 g, $4.48 \mathrm{mmol}, 56 \%)$.

$\mathrm{Mp} 111-113{ }^{\circ} \mathrm{C}$.

$[\alpha]_{D}^{25}-61.3\left(\mathrm{c}=1.015, \mathrm{CHCl}_{3}\right)$.

IR: $1743,1723 \mathrm{~cm}^{-1}$.

${ }^{1} \mathrm{H}$ NMR $\left(400 \mathrm{MHz}, \mathrm{CDCl}_{3}\right): \delta=4.32(1 \mathrm{H}, \mathrm{d}, J=11.5 \mathrm{~Hz}), 3.85(1 \mathrm{H}, \mathrm{d}, J=11.5 \mathrm{~Hz}), 3.82(3 \mathrm{H}$, s), $3.77(3 \mathrm{H}, \mathrm{s}), 3.28(3 \mathrm{H}, \mathrm{s}), 3.25(3 \mathrm{H}, \mathrm{s}), 1.42(3 \mathrm{H}, \mathrm{s}), 1.29(3 \mathrm{H}, \mathrm{s})$.

${ }^{13} \mathrm{C}$ NMR $\left(400 \mathrm{MHz}, \mathrm{CDCl}_{3}\right): \delta=168.6,167.2,100.4,98.2,76.0,59.5,53.2,52.8,50.4,48.2$, $17.8,17.7$

HRMS (+ESI): $m / z$ calcd for $\mathrm{C}_{12} \mathrm{H}_{20} \mathrm{NaO}_{8}\left(\mathrm{MNa}^{+}\right)$315.1056; found: 315.1058 . 


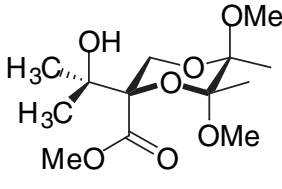

16

n-Butyllithium (1.9 mL, 1.6M in hexanes, $3.04 \mathrm{mmol})$ was added slowly to a stirred solution of diisopropylamine $(480 \mu \mathrm{L}, 3.42 \mathrm{mmol})$ in THF (3 $\mathrm{mL})$ at $-20{ }^{\circ} \mathrm{C}$ to $0{ }^{\circ} \mathrm{C}$. The solution was stirred for an additional $15 \mathrm{~min}$, then added via cannula to a stirred solution of 1 (477 $\mathrm{mg}, 2.04 \mathrm{mmol})$ in THF $(10 \mathrm{~mL})$ at $-78^{\circ} \mathrm{C}$. After $10 \mathrm{~min}$, acetone $(750 \mu \mathrm{L}, 10.09 \mathrm{mmol})$ was added. The reaction mixture was stirred for $1 \mathrm{~h}$, then quenched at -78

${ }^{\circ} \mathrm{C}$ with saturated $\mathrm{NH}_{4} \mathrm{Cl}$ and diluted with $\mathrm{Et}_{2} \mathrm{O}(30 \mathrm{~mL})$. The organic phase was washed with saturated $\mathrm{NH}_{4} \mathrm{Cl}(30 \mathrm{~mL})$ and water $(30 \mathrm{~mL})$, dried $\left(\mathrm{Na}_{2} \mathrm{SO}_{4}\right)$, filtered and concentrated in vacuo. The residue was purified by flash chromatography (hexane-EtOAc 4:1) to give $\mathbf{1 6}$ as a colourless oil (397 mg, $1.36 \mathrm{mmol}, 67 \%)$.

$[\alpha]_{\mathrm{D}}^{25}-124.0\left(\mathrm{c}=0.905, \mathrm{CHCl}_{3}\right)$.

IR: $3500,1731 \mathrm{~cm}^{-1}$.

${ }^{1} \mathrm{H}$ NMR $\left(400 \mathrm{MHz}, \mathrm{CDCl}_{3}\right): \delta=4.23(1 \mathrm{H}, \mathrm{d}, J=11.6 \mathrm{~Hz}), 4.02(1 \mathrm{H}, \mathrm{d}, J=11.6 \mathrm{~Hz}), 3.75(3 \mathrm{H}$, s), $3.233(3 \mathrm{H}, \mathrm{s}), 3.227(3 \mathrm{H}, \mathrm{s}), 2.96(1 \mathrm{H}, \mathrm{s}), 1.31(3 \mathrm{H}, \mathrm{s}), 1.24(3 \mathrm{H}, \mathrm{s}), 1.17(3 \mathrm{H}, \mathrm{s}), 1.09(3 \mathrm{H}, \mathrm{s})$.

${ }^{13} \mathrm{C}$ NMR (400 $\mathrm{MHz}, \mathrm{CDCl}_{3}$ ): $\delta=172.0,99.4,97.7,77.9,73.2,59.0,52.0,50.5,48.1,25.2,23.1$, 17.7, 17.6.

HRMS (+ESI): $m / z$ calcd for $\mathrm{C}_{13} \mathrm{H}_{24} \mathrm{NaO}_{7}\left(\mathrm{MNa}^{+}\right)$315.1420; found: 315.1413 .

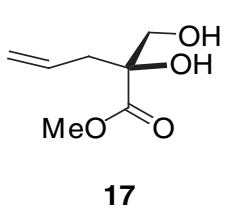

p-Toluenesulfonic acid monohydrate $(178 \mathrm{mg}, 0.93 \mathrm{mmol})$ was added to a stirred solution of 3 (128 mg, $0.46 \mathrm{mmol})$ in $\mathrm{MeOH}(10 \mathrm{~mL})$ under argon. The reaction mixture was refluxed for $2 \mathrm{~h}$, neutralized with $\mathrm{Et}_{3} \mathrm{~N}(130 \mu \mathrm{L}$, $0.93 \mathrm{mmol}$ ) and the solvent was removed under vacuo. The residue was purified by flash chromatography $\left(\mathrm{CH}_{2} \mathrm{Cl}_{2}\right.$-acetone $\left.9: 1\right)$ to give $\mathbf{1 7}$ as a colourless oil (70 mg, $0.43 \mathrm{mmol}, 94 \%)$.

$[\alpha]_{\mathrm{D}}^{25}-30.4\left(\mathrm{c}=0.705, \mathrm{CH}_{3} \mathrm{OH}\right)$.

IR: $3451,1732,1642 \mathrm{~cm}^{-1}$.

${ }^{1} \mathrm{H}$ NMR (400 MHz, CD $\left.{ }_{3} \mathrm{OD}\right): \delta=5.76-5.65(1 \mathrm{H}, \mathrm{m}), 5.02-4.93(2 \mathrm{H}, \mathrm{m}), 3.64(1 \mathrm{H}, \mathrm{d}, J=11.2$ $\mathrm{Hz}), 3.64(3 \mathrm{H}, \mathrm{s}), 3.63(1 \mathrm{H}, \mathrm{d}, J=11.2 \mathrm{~Hz}), 2.34(1 \mathrm{H}, \mathrm{dd}, J=14.0 \mathrm{~Hz}, 7.6 \mathrm{~Hz}), 2.25(1 \mathrm{H}, \mathrm{dd}, J=$ 14.0 Hz, $6.8 \mathrm{~Hz})$.

${ }^{13} \mathrm{C}$ NMR (400 MHz, $\left.\mathrm{CD}_{3} \mathrm{OD}\right): \delta=176.3,133.9,119.3,80.4,68.9,53.2,41.4$

HRMS (+ESI): $m / z$ calcd for $\mathrm{C}_{7} \mathrm{H}_{12} \mathrm{NaO}_{4}\left(\mathrm{MNa}^{+}\right)$183.0633; found: 183.0630 . 